\title{
Reabilitação feita com próteses parciais removíveis - relato de caso clínico
}

\author{
Rehabilitation done with removable partial dentures - clinical case report
}

Rehabilitación realizada con prótesis parciales removibles - reporte de caso clínico

\section{Stefani Duque Hermanson ${ }^{*}$, Athaluama Pires da Silva Inocencio ${ }^{2}$, Carla Minozzo Mello ${ }^{3}$, Oswaldo Luiz Cecilio Barbosa ${ }^{4}$, Carlos Roberto Teixeira Rodrigues ${ }^{5}$}

Como citar esse artigo. Hermanson SD; Inocencio, APS; Mello, CM; Barbosa, O.L.C; Rodrigues, CRT. Reabilitação feita com próteses parciais removíveis - relato de caso clínico. Revista PróUniverSUS. 2021 Jan./Jun.; 12 (1): 5053 .

\section{Resumo}

A procura por tratamento dentário estético vem aumentando consideravelmente no decorrer dos anos, ao qual muitos pacientes optam pela utilização de Próteses Parciais Removíveis. O objetivo deste artigo foi relatar, através de uma revisão de literatura e descrição de um caso clínico de reabilitação estética feita com próteses parciais removíveis com finalidade estética e funcional. Conclui-se que a reabilitação através de próteses parciais removíveis é uma excelente opção para tratamentos funcionais e estéticos e em alguns casos, para se manter a harmonia do sorriso, são necessários outros procedimentos estéticos como a restauração com resina composta.

Palavras-chave: Reabilitação; Prótese Parcial Removível; Estética.

\begin{abstract}
The search for aesthetic dental treatment has been increasing considerably over theyears, to which many patients choose the use of Removable Partial Prostheses. The aim of this article was to report, through a literature review and description of a clinical case of aesthetic rehabilitation made with removable partial prosthesis for aesthetic and functional purposes. It is concluded that rehabilitation through removable partial prostheses is an excellent option for functional and aesthetic treatments and in some cases, to maintain the harmony of the smile, other aesthetic procedures such as restoration with composite resin are necessary. Keywords:Removable Partial Prosthesis; Aesthetics.
\end{abstract}

\section{Resumen}

La demanda de tratamiento dental estético ha aumentado considerablemente a lo largo de los años, a lo que muchos pacientes optan por utilizar prótesis parciales extraíbles. El objetivo de este artículo era informar, a través de una revisión de la literatura y la descripción de un caso clínico de rehabilitación estética hecha con prótesis parciales extraíbles para fines estéticos y funcionales. Se concluye que la rehabilitación a través de prótesis parciales desmontables es una excelente opción para tratamientos funcionales y estéticos y en algunos casos, para mantener la armonía de la sonrisa, se necesitan otros procedimientos estéticos como la restauración con resina compuesta.

Palabras clave: Rehabilitación; Prótesis Parcial Extraíble; Estética.

\section{Afiliação dos autores:}

${ }^{1}$ Acadêmica no Curso de Odontologia, Universidade de Vassouras. Vassouras, RJ, Brasil. E-mail: stefaniduque@bol.com.brORCID: https://orcid.org/0000-0003-4875-4111

${ }^{2}$ Mestre. Professora do Curso de Odontologia, Universidade de Vassouras. Vassouras, RJ, Brasil. E-mail:athaluamapires@gmail.comORCID: https://orcid.org/0000-0001-5869-4357 ${ }^{3}$ Mestre. Professora do Curso de Odontologia, Universidade de Vassouras. Vassouras, RJ, Brasil. E-mail:carlaminozzo@uol.com.brORCID: https://orcid.org/0000-0002-7552-4154 ${ }^{4}$ Mestrando. Professor do Curso de Odontologia, Universidade de Vassouras. Vassouras, RJ, Brasil.E-mail:oswaldolcbarbosa@hotmail.comORCID: https://orcid.org/0000-00025668-3755

${ }^{5}$ Doutor. Professor do Curso de Odontologia, Universidade de Vassouras. Vassouras, RJ, Brasil. 


\section{Introdução}

A procura por tratamento dentário estético vem aumentando consideravelmente no decorrer dos anos ${ }^{1-2}$. Uma das questões mais comuns consiste na reabilitação de pacientes desdentados parcialmente, exigindo do profissional um diagnóstico preciso bem como um planejamento eficaz.

Existem inúmeras técnicas utilizadas na reabilitação de pacientes edêntulos como próteses e implantes $^{3}$. Nas últimas décadas houve uma redução do edentulismo, contudo ainda haverá demanda por Próteses Parciais Removíveis (PPR) por conta do aumento da expectativa de vida da população, outra questão que corrobora com essa demanda consiste nas diferenças socioeconômicas que são destoantes na nossa sociedade e que produzem realidades sociais extremamente diferentes, fazendo assim com que cada paciente tenha anseios, necessidades e expectativas bem particulares ${ }^{2}$. Apesar da conscientização quanto à higienização e manutenção dos elementos dentários devido ao enfoque que a saúde oral tem recebido nas últimas décadas, o edentulismo continuará a ser um problema pelo menos em médio prazo para a maioria dos pacientes, principalmente os idosos ${ }^{4-6}$.

O planejamento e a confecção de uma prótese exigem que o dentista se preocupe com diversas questões como função da articulação temporomandibular, saúde da mucosa oral, tonicidade da musculatura, distribuição das forças mastigatórias, higiene oral e da prótese, adaptação e extensão da prótese ${ }^{7}$. Dentre essas etapas uma que possui grande importância é a prova dos dentes da PPR, uma vez que nessa etapa é possível realizar diversos ajustes ${ }^{8}$. Mesmo a PPR sendo uma das técnicas mais utilizadas alguns problemas de cunho fonoaudiológico pode surgir por conta da utilização da mesma ${ }^{9-10}$. A Fonoaudiologia como parte da equipe interdisciplinar pode contribuirpara a adaptação protética e reabilitação das funções estomatognáticas dentro das limitações encontradas, de modo a proporcionar uma melhor qualidade de vida ao sujeito ${ }^{11}$.

Além das possíveis interferências na fala a PPR pode também mudar o modo como os pacientes mastigam $^{9-10}$. Quando ocorrem esses casos é necessário que haja uma atuação interdisciplinar entre dentistas e fonoaudiólogos ${ }^{9}$. Em muitos casos, para melhorar a estética do sorriso, é necessário realizar outros tratamentos como por exemplo a restauração de alguns dentes. Atualmente uma das técnicas mais utilizadas consiste na restauração com resina composta ${ }^{12-13}$.

O objetivo deste artigo foi relatar, através de uma revisão de literatura e descrição de um caso clínico, a reabilitação estética feita com próteses parciais removíveis com finalidade estética e funcional.
Metodologia

O presente trabalho foi autorizado no Comitê de Ética e Pesquisa, sobre o parecer número 4.126.079.

Paciente L.C.S., sexo feminino, 51 anos, compareceu à clínica odontológica da Universidade de Vassouras relatando não estar satisfeita com a estética dos seus dentes (Figura 1). Foi feita a anamnese com a paciente na qual relatou a perda dos dentes precocemente, por ser portadora de periodontite crônica, tornando-a parcialmente edêntula na arcada dentária superior e inferior (Figura 2). Após a anamnese realizou-se o exame clínico e solicitou-se uma radiografia panorâmica. Como exame radiográfico feito prosseguiu-se com a moldagem utilizando alginato na arcada superior e inferior. Com os modelos de gesso foi feito o planejamento e em seguida os preparos dos nichos nos dentes, logo após o paciente foi moldado novamente, confeccionando-se o modelo de gesso de ambas as arcadas dentárias e enviado ao laboratório de prótese dentária. Foram feitos os seguintes procedimentos clínicos: raspagem supragengival da arcada superior e inferior e profilaxia.

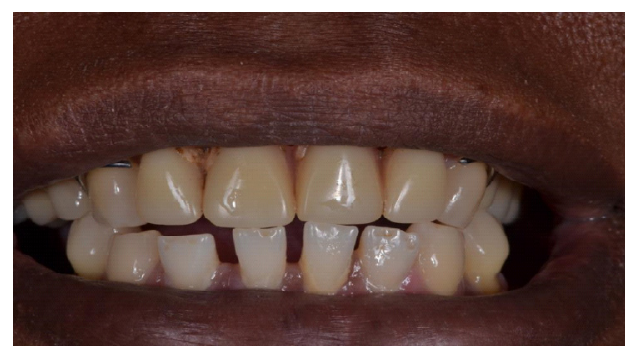

Figura 1. Aspecto inicial do sorriso da paciente.

Fonte: Autoria Própria

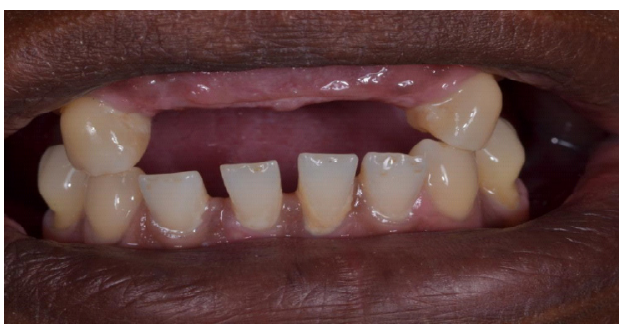

Figura 2. Aspecto inicial sem a prótese.

Fonte: Autoria Própria

Na prova da estrutura metálica fez-se a escolha da cor, forma e tamanho dos dentes e em seguida a moldagem funcional. Após a realização da prova da estrutura metálica encaminhou-se o modelo para o protético. Ao retornar do protético foi feita a prova dos dentes e escolha da cor da gengiva, sendo o trabalho enviado novamente para acrilização (Figura 3). 


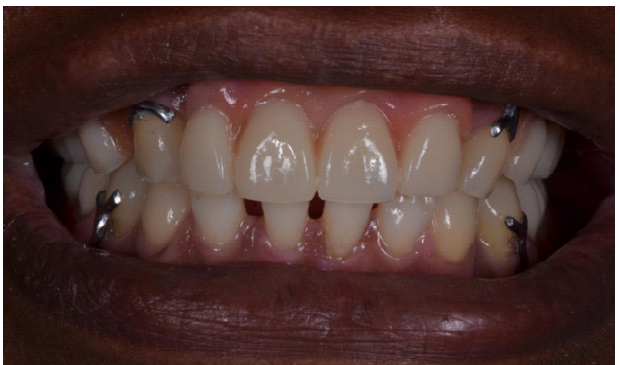

Figura 3. Aspecto clínico com a nova PPR.

Fonte: Autoria Própria

Realizou-se também restaurações com resina composta nos dentes 31,32 e 41 . Inicialmente fezse profilaxia dos dentes em questão (Figura 4) e seleção das cores de dentina e esmalte (Figura 5). O isolamento absoluto do campo operatório foi realizado utilizando lençol de borracha. Em seguida foi feito condicionamento ácido dos incisivos centrais e lateral, aplicação do adesivo e realizou-se a polimerização conforme instrução do fabricante pelas faces vestibular e lingual.

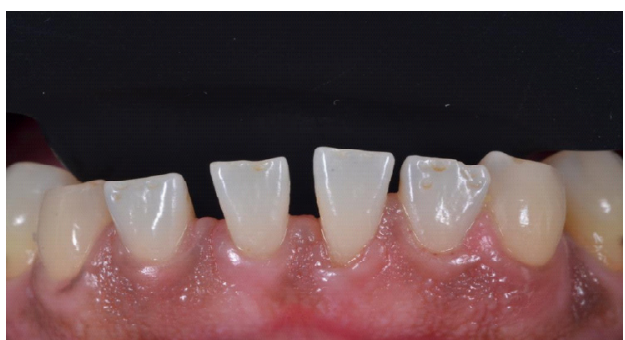

Figura 4. Dentes 31, 32 e 41 sem restauração.

Fonte: Autoria Própria

A resina composta foi aplicada para simular a cor da dentina na região cervical, terço médio e incisal. Após a polimerização desse incremento aplicou-se uma camada de resina composta para o esmalte sobre a superfície vestibular e espalhada com o auxílio de uma espátula e pincel sintético. Cada incremento foi polimerizado pelo tempo recomendado pelo fabricante, de forma contínua. Os mesmos procedimentos foram feitos em todos os dentes o qual necessitava do fechamento do diastema.

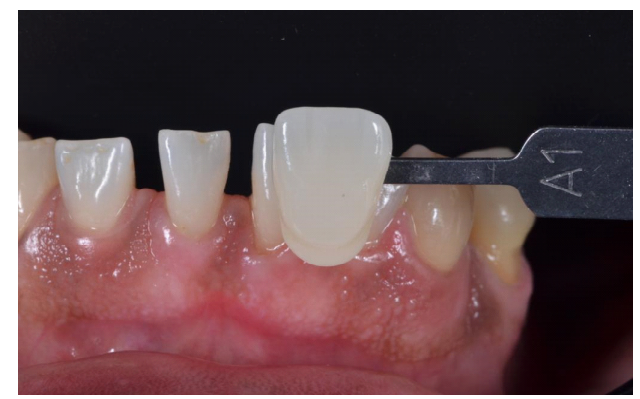

Figura 5. Seleção da cor dos dentes inferiores.

Fonte: Autoria Própria
Após a remoção do isolamento absoluto fez-se a remoção dos excessos e realizou-se o ajuste incisal e acabamento. O polimento da resina composta foi realizado uma semana após (Figura 6).

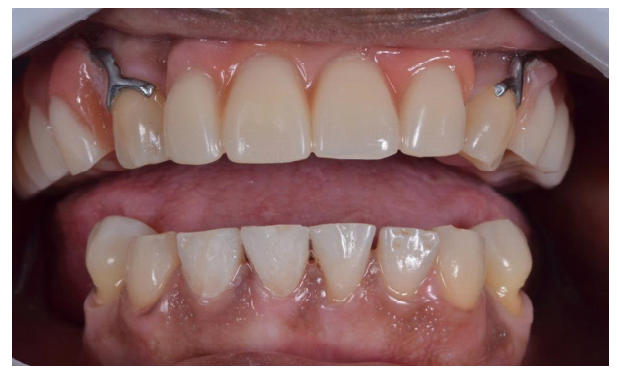

Figura 6. Aspecto final do sorriso da paciente após instalação da PPR e restaurações dos dentes inferiores.

Fonte: Autoria Própria

\section{Discussão}

O número de usuários de próteses removíveis vem aumento significativamente e junto a essa demanda há também a preocupação com a satisfação dos pacientes $1-2$.

O tratamento de reabilitação através da prótese pode ser realizado utilizando diversas técnicas tais como prótese removível ou fixa, parcial ou total, ou até mesmo por meio da utilização de tratamentos mais modernos, como por exemplo a prótese sobre implante, porém é importante ressaltar que todas essas técnicas são alternativas capazes de resolver o problema de perda dentária ${ }^{3}$. No que se refere ao caso clínico optou-se pela utilização de prótese parcial removível, que se mostrou uma excelente alternativa.

Uma questão importante na fase do tratamento reabilitador, constitui a prova estética e funcional da prótese que deve ser realizada juntamente com o paciente, uma vez que esse processo permite ter uma prévia muito fidedigna do resultado da prótese e caso algo não esteja de acordo é possível realizar alterações ${ }^{8}$. Tal procedimento, apontado pela literatura ${ }^{8}$, foi realizado também no caso clínico exposto, nesse procedimento foi possível realizar alguns ajustes como por exemplo a cor da gengiva.

Embora as próteses sejam as mais utilizadas na reabilitação dentária é importante observar que ela possui alguns contrapontos. Por exemplo, alguns autores ${ }^{9-1} 0$ relatam que as próteses dentárias podem afetar diretamente a fala, ao qual algumas questões fonoarticulatórias serem resultantes da reabilitação oral, sendo esse fato importante para o sucesso e satisfação do paciente com o tratamento odontológico. As próteses removíveis parciais ou totais podem também interferir na mastigação dos pacientes, fazendo com 
que a mastigação desses pacientes tenha alterações no corte dos alimentos bem como indefinição do tipo de mastigação ${ }^{9-12}$. A mastigação deve ocorrer após o corte do alimento com os incisivos, com lábios fechados, sem ruídos ou participação exagerada da musculatura perioral, com lateralização e mastigação bilateral alternada, com movimentos rotatórios de mandíbula e com simetria de força muscular ${ }^{14}$.

Sendo assim é necessário que seja feito um trabalho interdisciplinar entre a fonoaudiologia $\mathrm{e}$ odontologia9.

Já outros autores relatam o oposto, afirmando que as próteses parciais removíveis se incorporam ao sistema estomatognático e não interferem no equilíbrio biomecânico ${ }^{13}$. Mediante esse contexto é importante observar que inicialmente a paciente não demonstrou alterações na fala e nem demonstrou ter havido interferência no seu processo biomecânico.

Embora alguns pacientes optem pela utilização de próteses removíveis é importante ressaltar que outros procedimentos são necessários para que se tenha um sorriso aceitável esteticamente, por exemplo há a necessidade de se realizar restaurações de alguns dentes. Dentre os materiais utilizados para restauração a resina é uma das mais utilizadas, pois mesmo com a utilização da cerâmica essa ainda continua sendo um ótimo recurso, pois proporciona resultados estéticos satisfatórios ${ }^{15}$. As resinas compostas tiveram seu uso popularizado em dentes posteriores a partir do início dos anos 80, sendo reforçado pelo aprimoramento de suas características e técnica restauradora até os dias atuais $^{16-18}$.

Mais do que recuperar a função e a estética, a reabilitação de pacientes edêntulos é de suma importância porque permite que os pacientes recuperem sua autoestima ${ }^{5-19}$, resultando em melhora da sua qualidade de vida ${ }^{4-20}$. No caso clínico exposto fez-se uma reconstrução com resina composta para simular a cor da dentina procurando manter assim uma harmonia estética com os demais dentes e prótese.

\section{Considerações Finais}

Conclui-se que a reabilitação através de próteses parciais removíveis é uma excelente opção para tratamentos funcionais e estéticos e em alguns casos, para se manter a harmonia do sorriso, são necessários outros procedimentos estéticos como a restauração com resina composta.

No caso clínico apresentado no presente artigo, obteve-se um resultado estético e funcional satisfatório, pois demonstrou uma estética natural e funcional com excelentes características visuais, além de ter permitido uma grande interação entre a restauração e a prótese com o sorriso da paciente.

\section{Referências}

1. Areias C, Fonseca P, Figueiral MH. Satisfação dos pacientes portadores de prótese removível. JADA. 2007; 7(4): 49-53.

2. Farias Neto A, Carreiro AFP, Rizzatti-Barbosa CM. A Prótese parcial removível no contexto da odontologia atual. Odontol. Clín.-Cient. (Online) [online], 2011; 10(2): 125-128.

3. Costa EC, Fernandes Neto JÁ, Catão MHCV. Reabilitação oral em pacientes idosos revisão de literatura. In: Congresso Brasileiro de Ciências e da Saúde, 2016, Campina Grande. In: Anais I CONBRACIS. 2016; Campina Grande.

4. Koshino H, Hirai T, Ishijima T, Tsukagoshi H, Ishigami T, Tanaka Y.Quality of life and masticatory function in denture wearers. J Oral Rehabil. 2006; 33(5): 9-323.

5. Roumanas ED. The social solution-denture esthetics, phonetics, and function. J Prosthodont. 2009; 18(2): 5-112.

6. Vecchia MPD, Regis RR, Cunha TR, Andrade IM, Matta JCS, Souza RF. A randomized trial on simplified and conventional methods for complete denture fabrication: cost analysis. J Prosthodont. 2014; 23(3): 91-182.

7. Barbosa MT, Neto Izolani O, Rodrigues CRT, Laport LBR, Oliveira WS, Oliveira TBSE. Lesões bucais provocadas pelo uso de próteses removíveis. Brazilian Journal of Surgery and Clinical Research. 2018; 22(1) $62-66$

8. Goiato MC, Nobrega AS, Gennari Filho H, Santos DM. Prova Estética E Funcional - Uma Decisão Em Conjunto. Revista Odontológica de Araçatuba. 2014; 35(2): 09-14.

9. Olchik MR, Baltezan RL, Dornelles S, Levy DS, Reis Só MV, Santos RB, Ayres A, Presotto M. Alterações Miofuncionais em Adultos e Idosos Usuários de Prótese Dentária. Revista da Faculdadultfe de Odontologia de Porto Alegre2012; 53(5):6-11.

10. Rosa RR, Berretin-Felix G. Fala e Reabilitação oral protética: Revisão Integrativa Distúrbios da Comunicação. 2015; 27(3): 174-181.

11. Felício CM de, Cunha CC. Relações entre Condições Miofuncionais Orais e Adaptação de Próteses Totais. PCL 2005; 7(36): 195-202.

12. Cavalcanti RVA,Bianchini EMG. Verificação e análise morfofuncional das características da mastigação em usuários de prótese dentária removível. Rev. CEFAC [online]. 2008; 10(4): 490-502.

13. Meyer GA,Oliveira Júnior FB, Oliveira LV, Sousa BP, Rodrigues FV, Lima EMCX. Soluções Estéticas Para Próteses Parciais Removíveis Retidas Por Grampos Metálicos. Revista Bahiana de Odontologia. 2013; 4(2): 93 103

14. Medeiros AMC, Medeiros M. Motricidade Orofacial: Inter-relação entre Fonoaudiologia \& Odontologia. São Paulo: LOVISE, 2006.

15. Guerra MLRS, Venâncio GN, Augusto CR. Fechamento de diastemas anteriores com resina composta direta: relato de caso. FOL -Faculdade de Odontologia de Lins. 2017; 1(2): 317-322.

16. Anusavice KJ. Quality Evaluation of Dental Restorations: Criteria for placement and replacement. Quintessence; 1987.

17. Mair LH. Ten-year clinical assessment of three posterior resin composites and two amalgams. Quintessence International. 1998; 29(81): 90-483.

18. Debastiani FS, Lopes GC. Restaurações diretas de resinas compostas em dentes Posteriores. International Journal of Brazilian Dentistry. 2005; 1(1): 30-39.

19. Barbosa DB, Assunção WG, Gennari Filho H, Goiato MC. Instalação de Prótese Total: uma revisão. Revista de Odontologia da unesp. 2006; 35 : $53-60$

20. Goiato MC, dos Santos DM, Silva EVF. Como realizar o selamento periférico e a moldagem funcional? Revista Odontológica de Araçatuba. 2013; 34: 14-19. 